\title{
Protein Interaction and Transport Maps of Live Cell Nuclei Using Fluorescence Correlation Spectroscopy in a Single Plane Illumination Microscope
}

\author{
Jan W. Krieger ${ }^{1}$, Agata Pernus ${ }^{1}$, Peter Brazda ${ }^{2}$, Jörg Langowski $^{1}$
}

1 Division Biophysics of Macromolecules, German Cancer Research Center, Im Neuenheimer Feld 580, D-69120 Heidelberg, Germany

2 Department of Biochemistry and Molecular Biology, Immunogenomics Research Group, Research

Center for Molecular Medicine, University of Debrecen, Hungary

Proteins acting on DNA need to penetrate a dense network of chromatin and associated macromolecules in the cell nucleus to access their target sites. Intracellular mobility of proteins is characterized by diffusion coefficients of the order of $1-100 \mu \mathrm{m}^{2} / \mathrm{s}$, leading to millisecond time scales for movement on the submicrometer scale.

Typical microscopic methods used for characterizing intracellular protein mobility are, e.g., fluorescence photobleaching recovery (FRAP) and fluorescence correlation spectroscopy (FCS). Of these, FRAP can image protein mobility in entire two-dimensional sections of live cells, but is typically limited to the time resolution of confocal image series, some frames per second. FCS, on the other hand, has fast time resolution but so far has been limited to single-point measurements in the focus of a laser beam. Although we have demonstrated first protein mobility maps by point-to-point FCS (1), this method is extremely time-consuming and not very feasible for live cell measurements.

Here we show results from single plane illumination microscopy based fluorescence correlation spectroscopy (SPIM-FCS), a new method for imaging FCS in 3D samples that combines the fast time resolution of FCS with the possibility of acquiring the mobility data in parallel on an entire twodimensional cross-section (2). This will then provide diffusion coefficients, flow velocities and concentrations in an imaging mode. Extending this technique to two-color fluorescence cross-correlation spectroscopy (SPIM-FCCS) also allows one to measure molecular interactions in an imaging mode (3).

The feasibility of the method and its agreement with classical point-focus FCS was first demonstrated with some simple test systems: EGFP oligomers, EGFP-mRFP fusion proteins and fluorescently labeled DNA oligonucleotides. As a model system for protein-protein interactions and DNA binding we studied c-Fos and c-Jun, two components of the AP-1 transcription factor, which form a heterodimer and whose interaction and diffusion behavior we had studied by point-focus FCS earlier (4). Using c-Fos-EGFP and c-Jun-mRFP fusion proteins in HeLa cells, we show here mobility maps of the two proteins, and simultaneously recorded interaction maps that show heterodimer formation. We find that the regions of the cell nucleus where the proteins show strong dimerization strongly correlate with the regions of immobilization, i.e. DNA binding. This shows that dimerization is a prerequisite for target binding and that most of the dimerized transcription factor is also bound to DNA. 
In a second example, we investigated the dynamical changes in binding and mobility of the retinoid acid receptor (RAR-RXR) upon activation by ligand binding (5). Activation shifts the population of the fluorescently labeled receptor into a slower-moving state, both increasing the fraction of the receptor population in this state and decreasing its diffusion coefficient

In summary, SPIM-FC(C)S is a powerful new technique that establishes the diffusional mobility of fluorescent markers in live cells as a new imaging parameter.

(1) Dross N, Spriet C, Zwerger M, Muller G, Waldeck W, and Langowski J: Mapping eGFP oligomer mobility in living cell nuclei. PLoS One 4 (4), e5041, 2009.

(2) Singh AP, Krieger JW, Buchholz J, Charbon E, Langowski J, and Wohland T: The performance of 2D array detectors for light sheet based fluorescence correlation spectroscopy. Opt Express 21 (7), 8652-8668, 2013.

(3) Krieger JW, Singh AP, Garbe CS, Wohland T, and Langowski J: Dual-Color Fluorescence CrossCorrelation Spectroscopy on a Single Plane Illumination Microscope (SPIM-FCCS). Opt Express 22 (3), 2358-2375, 2014.

(4) Baudendistel N, Müller G, Waldeck W, Angel P, and Langowski J: Two-Hybrid Fluorescence Cross-Correlation Spectroscopy Detects Protein-Protein Interactions In Vivo. Chemphyschem 6 (5), 984-990, 2005.

(5) Brazda P, Krieger J, Daniel B, Jonas D, Szekeres T, Langowski J, Toth K, Nagy L, and Vamosi G: Ligand binding shifts highly mobile RXR to chromatin-bound state in a coactivator-dependent manner as revealed by single cell imaging. Mol Cell Biol 2014. 